\title{
Estadiamento inicial dos casos de câncer de mama e colo do útero em
}

\section{mulheres brasileiras}

\author{
Initial staging of breast and cervical cancer in Brazilian women \\ Luiz Claudio Santos Thuler ${ }^{1}$, Gulnar Azevedo Mendonça²
}

\section{RESUMO}

Objetivo: analisar a evolução temporal do estadiamento no momento do diagnóstico dos casos de câncer de mama e do colo do útero em mulheres atendidas pelo Sistema Único de Saúde (SUS). Métodos: em uma primeira etapa foram identificados os relatórios disponíveis contendo a descrição do estadiamento clínico inicial dos casos de câncer atendidos nos hospitais brasileiros. Considerando-se sua escassez e pouca representatividade, realizou-se uma segunda etapa na qual foi efetuada busca ativa de informações. Uma planilha foi enviada por via postal a todos $(\mathrm{n}=173)$ os Centros de Alta Complexidade em Oncologia (CACON) cadastrados pelo Ministério da Saúde para atendimento ao SUS solicitando informações para o período compreendido entre 1995 e 2002. Para a análise estatística foi utilizado o programa "R". Os resultados são apresentados como percentuais e boxplots. Resultados: na primeira etapa (1990-1994) foram identificadas informações de 18 hospitais, referentes a 7.458 pacientes com câncer de mama e 7.216 pacientes com câncer do colo do útero. A mediana de casos diagnosticados em estádio avançado (estádios III e IV) foi de 52,6 e 56,8\%, respectivamente. Na segunda etapa (1995-2002) foram obtidas informações de 89 hospitais e 7 serviços isolados de quimioterapia ou radioterapia, referentes a 43.442 casos de câncer de mama e 29.263 casos de câncer de colo do útero. A taxa de resposta, baseada na listagem inicial de CACONs, foi de 55\%. A mediana do percentual de pacientes em estádio avançado foi de 45,3\% para os casos de câncer de mama e de 45,5\% para os casos de câncer do colo do útero. Conclusões: no Brasil, poucos estudos analisaram as tendências temporais do estadiamento dos casos de câncer. Os dados obtidos a partir dos registros hospitalares de Câncer mostraram que, na última década, houve redução no percentual de casos de câncer de mama e do colo do útero em estádio avançado, o que pode indicar que nas regiões onde estes hospitais estão localizados houve melhora na detecção precoce destes tipos de câncer.

PALAVRAS-CHAVE: Neoplasias do colo uterino; Neoplasias mamárias; Estadiamento de neoplasias; Registros de doenças

\section{ABSTRACT}

Purpose: to analyze time trends in the clinical staging at the moment of diagnosis in patients with breast and cervix cancer based on data produced by the Brazilian Public Health System (SUS). Methods: in the first part of this study we identified the published documents describing clinical staging of patients at the moment of diagnosis. Considering their scarcity and poor representativity we conducted the second part of this study through an active search for information. A form was sent via regular mail to all cancer centers in the country $(n=173)$ requesting information about the tumor site and stage at diagnosis by year, in the period of 1995-2002. The statistical analysis was performed using the "R" statistical package. The results are reported as percentage and boxplots. Results: in the first part of the study (1990-1994) we described data from 18 hospitals concerning 7,458 patients with breast cancer and 7,216 patients with cervix cancer. The median of the percentage of cancers diagnosed at an advanced stage (stages III or IV) was 52.6 and $56.8 \%$, respectively. In the second part of the study (1995-2002) data were collected from 89 cancer hospitals and 7 chemotherapy or radiotherapy clinics. There was a total of 43,442 cases of breast cancer and 29,263 of cervix cancer. The response rate based on the potential contact list was $55 \%$. The median percentage of patients in advanced stage was $45.3 \%$ for breast cancer and $42.5 \%$ for cervix cancer. Conclusions: few studies have examined the time trends in staging of cancer at diagnosis in Brazilian hospitals. Data obtained from Hospital Cancer Registries showed that in the last decade there was a reduction in the percentage of cervix and breast cancer at the advanced stage. This reduction can be due to an improvement in early detection of these cancers.

KEYWORDS: Cervix neoplasms; Breast neoplasms; Neoplasm staging; Diseases registries

Coordenação Nacional de Prevenção e Vigilância - Instituto Nacional de Câncer - INCA - Rio de Janeiro (RJ) - Brasil.

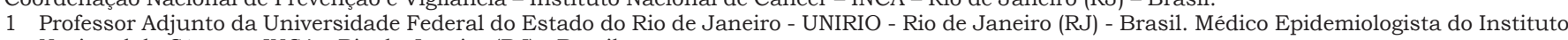
Nacional de Câncer - INCA - Rio de Janeiro (RJ) - Brasil.

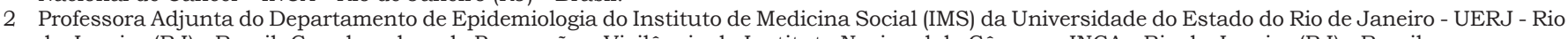
de Janeiro (RJ) - Brasil. Coordenadora de Prevenção e Vigilância do Instituto Nacional de Câncer - INCA - Rio de Janeiro (RJ) - Brasil.

Correspondência: Luiz Claudio Santos Thuler

Rua dos Inválidos, 212/4 andar - 20231020 - Rio de Janeiro - RJ - e-mail: 1thuler@inca.gov.br

Recebido em: 17/3/2005

Aceito com modificações em: 24/10/2005

Rev Bras Ginecol Obstet. 2005; 27(11): 656-60 


\section{Introdução}

As estimativas do Instituto Nacional de Câncer $^{1}$ apontam o câncer de mama e o câncer do colo do útero como as localizações de câncer com maior incidência entre as mulheres brasileiras. Além disso, enquanto nos países mais desenvolvidos as taxas padronizadas de mortalidade por esses cânceres vêm apresentando redução, no Brasil, no período compreendido entre 1979 e 2002, observouse incremento nas taxas de mortalidade por câncer de mama (de 8,57 para $11,18 / 100.000$ mulheres; variação percentual relativa $=+30 \%$ ) e constância nas taxas referentes ao câncer do colo do útero (de 4,97 para 5,03/100.000 mulheres; variação percentual relativa $=1 \%)^{2}$.

Tanto o câncer de mama quanto o câncer do colo do útero são considerados de bom prognóstico, se diagnosticados e tratados precocemente. Porém, o diagnóstico realizado em fase avançada da doença pode ser o maior responsável pela manutenção das taxas de mortalidade elevadas ${ }^{3}$, sugerindo que as pacientes não têm sido beneficiadas pelos procedimentos terapêuticos que podem de fato reverter o curso clínico da doença.

A avaliação do desempenho dos programas de controle dos cânceres de mama e do colo do útero deve ser feita por meio de indicadores, entre os quais está a descrição da evolução temporal dos percentuais de casos identificados em estádio avançado. Uma das principais fontes para esse tipo de informação são os Registros Hospitalares de Câncer (RHCs), que permitem, a partir dos dados coletados em sua rotina, aferir a qualidade da assistência hospitalar prestada ao paciente com neoplasia maligna, possibilitando avaliar protocolos terapêuticos e novas tecnologias, analisar objetivamente a extensão e evolução da doença, realizar estudos clínico-epidemiológicos e melhorar a qualidade das informações registradas no prontuário médico-hospitalar.

Este estudo teve como objetivo conhecer a extensão clínica do câncer de mama (CID $10=$ C50) e do câncer do colo do útero (CID $10=\mathrm{C} 53$ ), excluidos os tumores in situ (CID $10=$ D05 e D06, respectivamente), no momento do diagnóstico, em mulheres atendidas nos hospitais brasileiros que prestam serviço ao Sistema Único de Saúde (SUS).

\section{Método}

\section{Fonte de dados}

A partir dos arquivos do INCA e de uma consulta à internet usando a ferramenta de busca Google foi possivel identificar um pequeno número de relatórios de RHCs com dados sobre estadiamen- to clínico de pacientes, a maioria referentes ao período compreendido entre 1990 e 1994. A análise destes dados constituiu a primeira etapa do estudo.

Em função da baixa cobertura, falta de periodicidade e desatualização dos relatórios identificados, procedeu-se à segunda etapa do estudo, composta da busca ativa de informações. Para isso, encaminhou-se uma planilha por via postal a todos os Centros de Alta Complexidade em Oncologia (CACONs) cadastrados no Ministério da Saúde no ano de 2003 (n=173). Foram solicitadas informações sobre o número de casos de câncer de mama e do colo do útero matriculados anualmente no período compreendido entre 1995 e 2002, classificando-os conforme o estadiamento clínico no momento do diagnóstico. Os endereços dos hospitais foram obtidos na página do INCA em julho de 2003.

Foram excluídas as séries de casos nas quais os dados foram apresentados agrupados para todos os cânceres, impossibilitando a obtenção de dados individualizados sobre cada topografia. A Fundação Oncocentro de São Paulo (FOSP) apresentou uma planilha com dados anuais para os anos 2000, 2001 e 2002, referente aos CACONs que para lá encaminham seus dados rotineiramente.

\section{Variáveis incluidas no estudo}

A localização do tumor foi definida de acordo com a Classificação Estatística Internacional de Doenças e Problemas Relacionados à Saúde (CID 10), sendo consideradas as categorias C50 (câncer de mama) e C53 (câncer do colo do útero).

Para a definição do estadiamento foi usado o sistema proposto pela União Internacional Contra o Câncer - UICC, que se baseia em três componentes principais: características do tumor primário, características dos linfonodos das cadeias de drenagem linfática do órgão em que o tumor se localiza e presença ou ausência de metástases a distância. Nesta classificação o "T" representa o tamanho ou o volume do tumor primário, com subcategorias que variam de T0 a T4, o "N" indica a condição dos linfonodos regionais, com subcategorias que variam de NO a N3, expressando o nível de evolução do tumor e dos linfonodos comprometidos, e o " $\mathrm{M}$ " indica a presença ou ausência de metástases a distância, com as subcategorias $\mathrm{M} 1$ ou $\mathrm{M} 0$, respectivamente. O simbolo "X" é utilizado quando uma categoria não pode ser devidamente avaliada. Quando as categorias T, N e M são agrupadas em combinações pré-estabelecidas, formamse os estádios que, geralmente, variam de 0 a IV, com subclassificações A, B e C, em alguns estádios, para expressar o nível de evolução da doença ${ }^{4}$. Foram consideradas as seguintes categorias de estadiamento: estádio I, estádio II, estádio III, estádio IV e estádio ignorado. Segundo os critérios da Organização Mundial da Saúde, foram considerados em estádio avançado os casos com doença nos estádios III e IV ${ }^{5}$. 


\section{Análise dos dados}

As informações foram armazenadas como arquivo eletrônico no programa Excel (Microsoft Corporation, versão 5.1, 2002) e para análise estatística foi utilizado o programa "R" versão 1.8.1, 2003. Os cálculos tiveram por base os casos cujo estadiamento era conhecido. Os percentuais de casos em cada estádio foram apresentados agrupados para todo o periodo. Em seguida, os percentuais de casos em estádio avançado foram apresentados para cada ano, objetivando identificar possiveis tendências. Como o número de unidades informantes em cada um dos anos estudados foi variável, optouse por comparar graficamente a variabilidade destes últimos percentuais por meio de boxplots, visando destacar os valores mínimo e máximo, mediana, percentis 25 e 75 e outliers.

\section{Resultados}

Para a primeira etapa do estudo (1990 a 1994), foram identificadas informações de 18 hospitais, referentes a 7.458 casos de câncer de mama e 7.216 casos de câncer do colo do útero. O estadiamento inicial foi informado para 21,0 e $41,8 \%$ dos casos, respectivamente $^{6-17}$. A Tabela 1 apresenta a mediana da distribuição percentual dos estádios dos casos de câncer de mama e do colo do útero nesses hospitais. Observa-se que em mais da metade dos casos (52,5 e $56,8 \%$, respectivamente) o diagnóstico se fez quando a doença encontrava-se nos estádios avançados.

Tabela 1 - Distribuição percentual dos casos de câncer de mama e colo do útero segundo estadiamento inicial. Brasil, 1990 a 19946-17

\begin{tabular}{lrc}
\hline Variáveis & $\begin{array}{c}\text { Câncer de } \\
\text { mama }\end{array}$ & $\begin{array}{c}\text { Câncer do } \\
\text { colo do útero }\end{array}$ \\
\hline Total de casos & 7.458 & 7.216 \\
Casos com estádio ignorado, \% & 21,0 & 41,8 \\
Casos com estádio conhecido & 5.891 & 4.203 \\
Estádio I, \% mediano & 14,7 & 4,5 \\
Estádio II, \% mediano & 32,9 & 35,8 \\
Estádio III, \% mediano & 46,8 & 40,7 \\
Estádio IV, \% mediano & 3,9 & 18,0 \\
Estádio avançado (III e IV), \% mediano & 52,5 & 56,8 \\
\hline
\end{tabular}

$\mathrm{Na}$ segunda etapa deste estudo (1995 a 2002), a estratégia de busca ativa permitiu obter informações referentes a 89 hospitais e 7 serviços isolados de quimioterapia ou radioterapia vinculados a um CACON. A taxa de resposta, baseada na listagem inicial, foi de $55 \%$, variando de $10 \%$ na região Sul a $67 \%$ na região Sudeste. Considerando-se que em dezembro de 2002 o INCA apontava a existência de 103 RHCs implantados no país ${ }^{18}$, o número de hospitais incluídos no estudo equivale a $86 \%$ do número de hospitais com RHC. Nenhum hospital apresentou a série completa para os oito anos a que se referia a pesquisa.

Foram incluídos nesta etapa 43.442 casos de câncer de mama e 29.263 casos de câncer de colo do útero. O estadiamento inicial foi informado para 80,5 e $78,9 \%$ dos casos, respectivamente. A Tabela 2 apresenta a mediana da distribuição percentual dos estádios nos hospitais analisados. A maioria dos casos de câncer de mama foi diagnosticada no estádio II $(42,8 \%)$, ao passo que para o câncer do colo do útero predominou o estádio III $(37,7 \%)$. Os casos com estádio ignorado representaram cerca de $20 \%$ da casuística analisada, ao passo que os casos em estádio avançado corresponderam a $45 \%$ dos casos com estádio conhecido para ambas as localizações.

Tabela 2 - Distribuição percentual dos casos de câncer de mama e colo do útero segundo estadiamento inicial. Brasil, 1995 a 2002.

\begin{tabular}{lrc}
\hline Variáveis & $\begin{array}{c}\text { Câncer de } \\
\text { mama }\end{array}$ & $\begin{array}{r}\text { Câncer do } \\
\text { colo do útero }\end{array}$ \\
\hline Total de Casos & 43.442 & 29.263 \\
Casos com estádio ignorado, \% & 19,5 & 21,1 \\
Casos com estádio conhecido & 34.988 & 23.082 \\
Estádio I, \% mediano & 10,9 & 17,7 \\
Estádio II, \% mediano & 42,8 & 33,3 \\
Estádio III, \% mediano & 32,6 & 37,7 \\
Estádio IV, \% mediano & 12,3 & 6,1 \\
Estádio avançado (III e IV), \% mediano & 45,3 & 45,5 \\
\hline Fontes: dados primários. & &
\end{tabular}

Fontes: dados primários.

Por outro lado, conforme apresentado na Figura 1, considerando o valor mediano da distribuição percentual de casos de câncer de mama cujo diagnóstico ocorreu em estádio avançado (estádios III e IV), observa-se uma discreta redução ao longo dos últimos anos, passando de $50,6 \%$ dos casos em 1995 para 45,3\% em 2002. Em relação ao câncer do colo do útero, esse valor apresenta uma diminuição semelhante no mesmo período, passando de 51,7\% em 1995 para 42,5\% em 2002 (Figura 2).

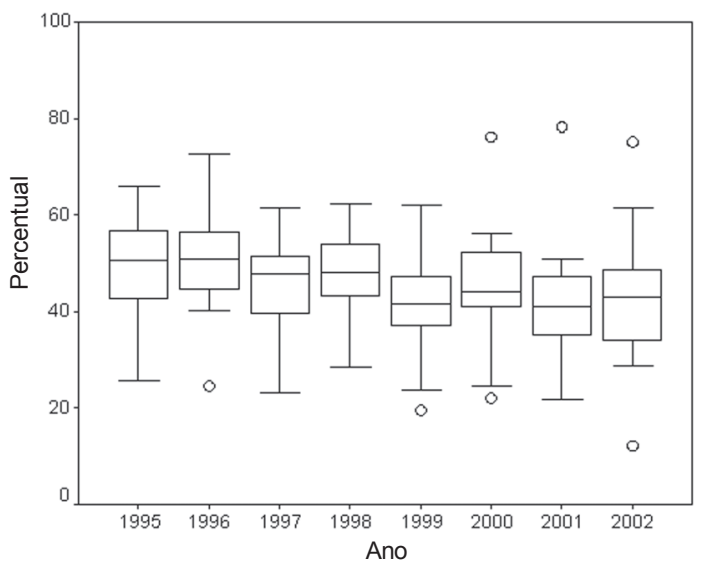

Obs: 0 boxplot apresenta os valores mínimo, máximo e os percentis 25,50 (mediana) e 75 do percentual de casos diagnosticados em estádio inicial por ano.

Figura 1 - Distribuição percentual dos casos de câncer de mama diagnosticados em estádios avançados da doença (III e IV). Brasil, 1995 a 2002. 


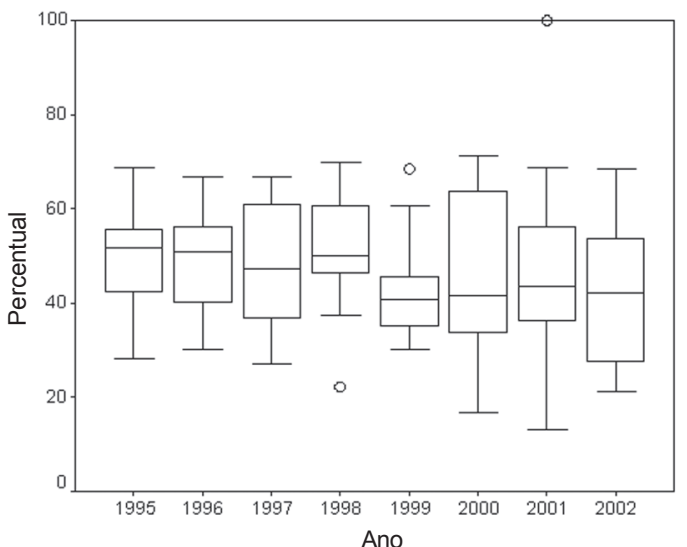

Obs: 0 boxplot apresenta os valores mínimo, máximo e os percentis 25, 50 (mediana) e 75 do percentual de casos diagnosticados em estádio inicial por ano.

Figura 2 - Distribuição percentual dos casos de câncer do colo do útero diagnosticados em estádios avançados da doença (estádios III e IV). Brasil, 1995 a 2002.

\section{Discussão}

Este estudo constitui um esforço para consolidar as informações referentes ao estadiamento inicial de pacientes com câncer de mama e do colo do útero atendidas pelo SUS com o objetivo de avaliar sua evolução temporal. O número de hospitais avaliados equivale a $86 \%$ dos hospitais com RHCs existentes no país. Entretanto, deve-se ressaltar que nenhum hospital apresentou informações para todos os oito anos a que se referia a pesquisa, o que deve ser considerado como uma limitação do estudo. Outra possivel limitação dos nossos resultados refere-se ao fato de que a implantação dos RHCs é um dos indicadores de qualidade dos CACONs. Assim sendo, os casos incluídos neste estudo podem refletir a realidade dos centros melhor qualificados para a atenção oncológica, podendo-se supor que o percentual de casos cujo diagnóstico é realizado em estádio avançado da doença seja ainda maior que o por nós descrito.

Ao confrontar os resultados obtidos na primeira fase do estudo (1990-1994) àqueles obtidos por busca ativa (1995-2002), observa-se que, para ambas as localizações topográficas, o valor mediano do percentual de pacientes em estádio avançado vem sofrendo queda progressiva. Em relação ao câncer de mama, os percentuais passaram de 52,5 para $45,3 \%$, e para o câncer do colo do útero, de 56,8 para $45,5 \%$.

Apesar dessa redução, os percentuais por nós observados são muito superiores àqueles observados em países desenvolvidos. Nos Estados Unidos, Liu et al. ${ }^{19}$ avaliaram os efeitos do programa nacional de detecção precoce dos cânceres de mama e colo do útero dirigido a mulheres de baixa renda e encontraram que, entre 1995 e 1999, o percentual de casos avançados de câncer de mama foi de 12,1 entre as mulheres incluídas no referido programa, ao passo que nas demais esse valor foi de 8,5. Em outro estudo realizado no mesmo país, Barry e Breen $^{20}$ analisaram mais de 12.000 casos de cân- cer de mama e observaram que apenas 7,9\% das mulheres foram diagnosticadas nos estádios IIIB ou IV. Por outro lado, Fregene e Newman ${ }^{21}$, ao sintetizar os dados sobre a ocorrência do câncer de mama entre as mulheres subsaarianas, referem que, naqueles países, o diagnóstico é realizado nos estádios III ou IV em 70 a $90 \%$ dos casos, valores muito superiores àqueles observados em nosso estudo.

Para o câncer do colo do útero observa-se situação semelhante àquela observada para o câncer de mama. Estudo realizado nos Estados Unidos da América, com dados de sete planos de saúde, mostrou que, no momento do diagnóstico, das 833 pacientes diagnosticadas com câncer do colo do útero entre 1995 e $2000,65 \%$ apresentavam doença localizada, 25\% apresentavam doença regional e somente em $7 \%$ foram observadas metástases a distân$\mathrm{cia}^{22}$. Por outro lado, estudo realizado na Inglaterra $^{23}$ mostrou que $23,8 \%$ dos 382 casos de câncer do colo do útero registrados entre 1985 e 1996 foram detectados nos estádios III e IV. Neste estudo 90\% dos casos detectados pelo rastreamento encontravam-se no estádio I. Situação semelhante à por nós descrita foi observada na Tunísia, onde $42,5 \%$ dos 216 casos novos de câncer do colo do útero diagnosticados em 1994 encontravam-se nos estádios III e $\mathrm{IV}^{24}$. Por outro lado, estudo realizado na Nigéria ${ }^{25}$ mostrou que em $3 / 4$ dos 169 pacientes com câncer do colo do útero diagnosticados entre 1990 a 1999 a doença encontrava-se em estádio avançado, valores muito superiores aos por nós descritos.

No Brasil, no que diz respeito ao câncer de mama, nos últimos anos tem-se observado aumento nas taxas de mortalidade, o que vem sendo atribuido, principalmente, a retardamento no diagnóstico e na instituição de terapêutica adequa$\mathrm{da}^{26}$. Considerando-se que os resultados desta pesquisa indicam que o estadiamento no momento do diagnóstico vem sendo feito cada vez mais precocemente, é justo especular que as intervenções terapêuticas não estejam sendo oferecidas de maneira oportuna para todas as pacientes, uma vez que não estão sendo capazes de interferir no padrão de mortalidade por esse câncer.

Já em relação ao câncer do colo do útero, podese atribuir a queda no percentual de pacientes em estádio avançado, em parte, ao diagnóstico mais precoce, conseqüência da ampliação do acesso ao exame preventivo de Papanicolaou ocorrida nos últimos anos. Até 1998 eram realizados pelo SUS, em média, 6,3 milhões de exames por ano; entre 1998 e 2002, periodo em que foram realizadas duas campanhas nacionais visando aumentar a oferta de exames na rede pública, esse número passou para 9,2 milhões; já em 2003 e 2004 foram realizados 10,4 milhões de exames por ano ${ }^{27}$. Entretanto, apesar desse aumento no número de exames, as ações de controle do câncer do colo do útero não estão sendo capazes de interferir no padrão de mortalidade pela 
doença, o que indica que a resolutividade do tratamento para os casos diagnosticados mais precocemente ainda está aquém do que se poderia esperar.

Levando-se em consideração as limitações deste estudo, nos últimos anos houve aparente redução no percentual de pacientes com câncer de mama e colo do útero com a doença em estádio avançado no momento do diagnóstico. Sendo assim, são necessários outros estudos que possam investigar de forma mais precisa esta observação. Vale salientar a importância de se garantir a cobertura e qualidade das informações produzidas pelos RHCs de forma que possam oferecer ferramentas essenciais para a gestão da atenção em câncer.

\section{Agradecimentos}

Fernanda Souza de Almeida, acadêmica da Faculdade de Estatística da UERJ e aos 89 hospitais e 7 serviços isolados de quimioterapia ou radioterapia participantes.

\section{Referências}

1. Brasil. Ministério da Saúde. Instituto Nacional de Câncer. Estimativas 2005. Incidência de Câncer no Brasil. Brasília: INCA; 2005.

2. Brasil. Ministério da Saúde. Instituto Nacional de Câncer. Divisão de Informação. Atlas de mortalidade por câncer no Brasil: 1979-1999. Brasília: INCA; 2002.

3. World Health Organization. National Cancer Control Programmes. Policies and managerial guidelines. $2^{\text {nd }}$ ed. Geneva: WHO; 2002.

4. Organização Mundial da Saúde. Agência Internacional de Registros de Câncer (IARC). Associação Internacional de Registros de Câncer (AIRC). Registro de câncer: princípios e métodos. Rio de Janeiro: INCA; 1995. (Publicação Científica, 95).

5. World Health Organization. National Cancer Control Programmes. Policies and managerial guidelines. $2^{\text {nd }}$ ed. Geneva: WHO; 1995.

6. Fundação Oncocentro de São Paulo. Registro hospitalar de câncer. São Paulo: A Fundação; 1996.

7. Fundação Antonio Prudente. Hospital A. C. Camargo. Centro de Pesquisas. Registro hospitalar de câncer: estatística 1994. Rio de Janeiro: INCA; 1997.

8. Liga Bahiana Contra o Câncer. Hospital Aristides Maltez. Registro hospitalar de câncer. Dados do Hospital Aristides Maltez 1991-1994. Salvador: LBCC; 1995.

9. Liga Bahiana Contra o Câncer. Hospital Aristides Maltez. Registro hospitalar de câncer. Dados do Hospital Aristides Maltez 1995. Salvador: LBCC; 1995.

10. Liga Paranaense de Combate ao Câncer. Registros de Câncer. Estudo da Sobrevida de 5.195 casos de câncer tratados no Hospital Erasto Gaertner no período de 1990 a 1992, e seguidos por 5 anos. Curitiba: LPCC; 1998.

11. Irmandade da Santa Casa de Misericórdia de Porto Alegre. Hospital Santa Rita. Registro Hospitalar de Câncer. Registro hospitalar de câncer 1992/1993: relatório dos dados referentes ao período de 01 de janeiro de 1992 a 31 de dezembro de 1993. Rio de Janeiro: INCA; 1997.

12. Irmandade da Santa Casa de Misericórdia de Porto Alegre. Hospital Santa Rita. Registro Hospitalar de Câncer. Registro hospitalar de câncer: dados 19941996. Rio de Janeiro: INCA; Ano?

13. Hospital São Marcos. Serviço de Registro Hospitalar de Câncer. Dados estatísticos do Hospital São Marcos. Teresina: HSM; 1993.

14. Brasil. Ministério da Saúde. Instituto Nacional de Câncer. Pró-Onco. Registro hospitalar de câncer do INCA. Rio de Janeiro: PRÓ-ONCO; 1991.

15. Brasil. Ministério da Saúde. Instituto Nacional de Câncer. Registro hospitalar de câncer: dados das unidades hospitalares do INCA - Hospital do Câncer, Hospital de Oncologia, Hospital Luiza Gomes de Lemos - 1992. Rio de Janeiro: Pró-Onco; 1994.

16. Ministério da Saúde. Instituto Nacional de Câncer. Registro hospitalar de câncer: dados das unidades hospitalares do INCA - relatório anual 1993. Rio de Janeiro: INCA; 2000.

17. Instituto do Câncer do Ceará. Registro Hospitalar de Câncer. Dados relativos ao período 1992/1996. Fortaleza: ICC; 1999.

18. Ministério da Saúde. Instituto Nacional do Câncer [homepage da Internet]. Relatório anual. 2002 [acesso 18 jul 2004]. Disponivel em: http://www.inca.gov.br

19. Liu MJ, Hawk H, Gershman ST, Smith SM, Karacek $\mathrm{R}$, Woodford ML, et al. The effects of a National Breast and Cervical Cancer Early Detection Program on social disparities in breast cancer diagnosis and treatment in Massachusetts. Cancer Causes Control. 2005;16(1):27-33.

20. Barry J, Breen N. The importance of place of residence in predicting late-stage diagnosis of breast or cervical cancer. Health Place. 2005;11(1):15-29.

21. Fregene A, Newman LA. Breast cancer in sub-Saharan Africa: how does it relate to breast cancer in AfricanAmerican women? Cancer. 2005;103(8):1540-50.

22. Leyden WA, Manos MM, Geiger AM, Weinmann S, Mouchawar J, Bischoff $\mathrm{K}$, et al. Cervical cancer in women with comprehensive health care access: attributable factors in the screening process. J Natl Cancer Inst. 2005;97(9):675-83.

23. Herbert A, Singh N, Smith JA. Adenocarcinoma of the uterine cervix compared with squamous cell carcinoma: a 12-year study in Southampton and South-west Hampshire. Cytopathology. 2001;12(1):26-36.

24. Maalej M, Mrad K, Kochbati L, Guigua A, Ben Abdallah M, Ben Ayed F, et al. Cervical cancer in Tunisia: an epidemiological, clinical and pathological study. Eur J Obstet Gynecol Reprod Biol. 2004;113(2):226-8.

25. Ijaiya MA, Aboyeji PA, Buhari MO. Cancer of the cervix in Ilorin, Nigeria. West Afr J Med. 2004;23(4):319-22.

26. Brasil. Ministério da Saúde. Instituto Nacional do Câncer. Controle do câncer de mama. Documento de consenso. Rev Bras Cancerol. 2004;50(2):77-90.

27. Ministério da Saúde. DATASUS [sítio da Internet]. Informações de Saúde. 2004 [citado 2004 Jul 18]. Disponivel em: http://www.datasus.gov.br 